- Original Article

\title{
The Effect of Education Based on the Theory of Planned Behavior on Iron Supplementation among Pregnant Women
}

\author{
Zeinab Jalambadani ${ }^{1, *}$, Abasalt Borji' ${ }^{2}$, Mohammadbagher Delkhosh ${ }^{3}$ \\ 'Department of Public Health, Neyshabur University of Medical Sciences, Neyshabur, Iran \\ ${ }^{2}$ Department of Basic Medical Sciences, Neyshabur University of Medical Sciences, Neyshabur, Iran \\ ${ }^{3}$ Department of Occupational Health Engineering, Neyshabur University of Medical Sciences, Neyshabur, Iran
}

Background: Iron is an essential element for women of reproductive age, especially in the period before and during the pregnancy. This study investigates the consumption of iron to prevent iron deficiency anemia among pregnant women visiting Neyshabur healthcare centers based on the theory of planned behavior.

Methods: In this experimental study, data were collected through a questionnaire survey. It included 160 pregnant women who were receiving maternity services at twelve healthcare centers in the city of Neyshabur in Iran between 2015 and 2017. The participants' demographic and anthropometric characteristics, Using the theory of planned behavior, and blood lab examination results, including ferritin levels were measured and the data were analyzed using IBM SPSS ver. 22.0 (IBM Corp., Armonk, NY, USA).

Results: The average scores of knowledge, attitude, perceived behavioral control, subjective norms, and intention categories for the intervention group were meaningfully increased after the participants received education on iron supplementation $(\mathrm{P}<0.05)$. However, these changes were not found to be significant in the control group $(\mathrm{P}>0.05)$. No statistically significant difference was obtained in the subjective norms category between the two groups after the education intervention $(\mathrm{P}=0.92)$.

Conclusion: Based on the experimental effects of education encouraging iron supplementation in pregnant women, it is suggested that workshops promoting iron supplementation should be conducted in health centers with the aim of preventing widespread iron deficiency anemia.

Keywords: Anemia; Pregnant Women; Education

Received: December 27, 2017, Revised: February 14, 2018, Accepted: February 21, 2018

${ }^{*}$ Corresponding Author: Zeinab Jalambadani https://orcid.org/0000-0003-0803-7679

Tel: +98-433444011, Fax: +98-43334080, E-mail: jalambadaniz@gmail.com 


\section{INTRODUCTION}

The need for supplemental iron consumption during pregnancy has been well established. Because anemia in pregnancy is associated with increased rates of maternal and infant perinatal mortality, premature delivery, low birth weight, and other adverse outcomes, ${ }^{1)}$ the United States Preventive Services Task Force has recommended daily intake of iron supplements by pregnant women whose blood work shows a hemoglobin concentration $\geq 100 \mathrm{~g} / \mathrm{L}$. ${ }^{2)}$ According to a World Health Organization (WHO) report, ${ }^{3)}$ the global prevalence of anemia among pregnant women is $55.9 \% .^{4)} \mathrm{A}$ WHO report was also used to examine the prevalence of anemia in Iran in 2002 and he prevalence of anemia in children under six years of age was found to be $35 \%, 40 \%$ in pregnant women, and 33\% in non-pregnant women. ${ }^{4}$

Two studies carried out in Tehran, Iran's capital city, in 2002 and 2003 reported the prevalence of anemia among pregnant women as $14.9 \%$ and $8.6 \%$, respectively. ${ }^{5}$ The incidence of anemia ranging from $5 \%$ to $19.9 \%$ in a given population is considered to be a mild general health problem, and it is considered to be a moderate health problem if the incidence ranges from $20 \%$ to $39.9 \%$. If the prevalence of anemia is more than $40 \%$, this is considered a serious health concern. ${ }^{6)}$ Iron deficiency anemia has a harmful effect on mother and fetus, and it can increase rates of spontaneous abortion, early delivery, low birth weight, and fetal mortality. ${ }^{7)}$ Establishing a suitable model for behavior change is a priority for having an effective and effective intervention. The theory that has been chosen in this study to improve the use of iron supplementations in pregnant women is the theory of planned behavior that has been used in many studies, including healthy behaviors training, family planning, breast cancer, multivitamin use. ${ }^{8)}$ The theory of planned behavior was developed by Icek Azjen and his colleague Martin Fishbein, and it is categorized by the following:, ${ }^{90)}$ (1) attitude: a positive or negative evaluation of behavior that is based on behavioral beliefs and an assessment of behavior outcomes and attitudes toward behavior; (2) subjective norms: an evaluation based on the assumption that individuals are subjected to different social influences such as that from parents, a spouse, religious leaders, and so on; (3) behavioral intention: the decision and the will of the individual to behave in a particular manner; and (4) behavior: an individuals observable response in a given situation with respect to a given target.

The purpose of this study was to investigate the effect of education based on the theory of planned behavior in order to improve the use of iron supplementations and prevention of iron deficiency anemia among pregnant women referred to Neyshabur urban health centers. $^{11)}$

\section{METHODS}

An experimental design was employed to create a study of pregnant women from Neyshabur, Iran, between March 2015 and 2017. At the time of the study, there were 57 healthcare centers in the Neyshabur. Twelve of the centers were randomly selected as places to recruit par- ticipants. The women were randomly assigned to either the intervention group or the control group.

The criteria for participants included women between the ages of 18-49 years who were receiving midwifery services at local health centers, married and living with a spouse, and pregnancy at a gestational age of 16 weeks. Exclusion criteria included lack of willingness to participate in the educational sessions and a transfer to a different health care center.

Ethical approval for this study was gained from the research ethics committee at Tehran University of Medical Sciences (approval no., IR.TUMS.REC.1394.760) in 2015. Before enrolment, an informed consent was obtained from all of the participants.

\section{Sample Size}

The sample size was calculated using the formula for an experimental survey. ${ }^{12)}$ With a margin of error $=0.05$ and $10 \%$, an expected power of $90 \%, \mathrm{Z}=1.28$. The mean standard deviation (SD) in the intervention group (39.92 \pm 4.22$)$ and in the control group (36.26 \pm 4.77$)$ was calculated for a sample of 80 women in each of the two groups. Participants were pregnant women visitors to Neyshabur healthcare centers.

$$
n=\frac{\left(\mathrm{z}_{1-\alpha / 2}+\mathrm{Z}_{1-\beta}\right)^{2}\left(\partial_{1}{ }^{2}+\partial_{2}{ }^{2}\right)}{\left(\mu_{1}-\mu_{2}\right) 2}
$$

\section{Data Collection}

The data collection tool was a questionnaire consisting of the following categories: demographic information (13 items), knowledge (21 items), and questions covering the theory of planned behavior, including attitude (12 items), subjective norms (eight items), instructive behavior control (six items), behavioral intention (three items), and behavior (three items). For the 21 knowledge questions, the minimum knowledge score was zero and the maximum score was 21 . The values of the alpha coefficient were 0.84 and 0.90 , respectively. The attitude category included 12 questions with a response scale of opt-out (score 1) to highly agree (score 5). The value of the alpha coefficient was 0.85 and reliability was 0.97 . The subjective norm questions were measured with a 5 -point scale of 1 (disagree very much) to 5 (agree very much). The alpha coefficient was 0.71 and reliability was 0.96 . The control of perceived behavior was measured with a 5-point scale from 1 (very likely) to 5 (very low probability), which had an alpha of 0.79 and a margin of 0.95. Behavioral intention included a response scale with five options which ranged from very much opposite (score 1) to highly agree (score 5). The alpha coefficient was 0.80 and the reliability was 0.93. The behavior questions were answered with a response scale ranging from highly opposed (score 1) to highly agreeable (score 5). The alpha coefficient was 0.85 and the reliability was 0.93 .

The questionnaires were completed by the participants before the training presentation. Serum ferritin levels were also measured and recorded. Then, the participants were rated based on the score for each of the educational categories and according to the demographic variables. They were chosen and divided into an intervention group 
Table 1. Educational intervention based on theory of planned behavior

\begin{tabular}{|c|c|c|}
\hline Sessions & Educational content & Method of training \\
\hline First & $\begin{array}{l}\text { Discussed the benefits of iron supplementation. } \\
\text { Discussion and exchange of ideas to determine positive opinions and } \\
\text { attitudes of individuals to indirectly provide positive motivation to talk to } \\
\text { their spouses, develop new attitudes, and change any negative attitudes } \\
\text { toward sex during pregnancy. }\end{array}$ & $\begin{array}{l}\text { Lecture-group discussion-role playing. } \\
\text { Readings: the booklet includes information about the side effects and } \\
\text { benefits of taking iron supplements. } \\
\text { Two educational leaflets which include information on iron deficiency } \\
\text { and its effect on the fetus. }\end{array}$ \\
\hline Second & $\begin{array}{l}\text { Discussed the effect of iron supplementation on the fetus, the side effects } \\
\text { and benefits of iron supplementation during pregnancy, the best time for } \\
\text { pregnant women to take iron supplements, and proper nutrition and the } \\
\text { use of three types of foods to prevent iron deficiency. } \\
\text { Discussion ( } 30 \text { min) with women on intercourse were followed by an hour- } \\
\text { long discussion about common beliefs and misconceptions regarding iron } \\
\text { supplementation to help reduce misconceptions and to prepare the ground } \\
\text { for attitude and changes in beliefs about supplementation. }\end{array}$ & \\
\hline Third and fourth & $\begin{array}{l}\text { Discussion on the effect of taking iron supplements on the fetal development, } \\
\text { and the effects of iron deficiency on the fetus and the mother. What factors } \\
\text { interfere with iron absorption? } \\
\text { What is the best way and the best time to take iron supplements? Which } \\
\text { foods and other factors help with the body's absorption of? How can the } \\
\text { negative effects of poverty on iron supplementation be prevented? Does } \\
\text { high iron intake have side effects? }\end{array}$ & \\
\hline
\end{tabular}

$(\mathrm{n}=80)$ and a control group $(\mathrm{n}=80)$ by random allocation. The intervention group was exposed to four educational sessions of 75 minutes each, conducted in Neyshabur health centers in June 2017.

\section{Intervention}

The educational sessions for the intervention group were conducted by a health education expert. The control group received educational pamphlets. At 6 months post-intervention, follow-up questionnaires were administered to the intervention and control groups a second time. The details of the educational sessions are explained in Table 1.

\section{Statistical Analysis}

Data were analyzed using the IBM SPSS software ver. 22.0 (IBM Corp., Armonk, NY, USA), the Wilcoxon and Mann-Whitney non-parametric statistical tests, an independent t-test, and a chi-square test (significance level $\mathrm{P}<0.05$ ).

\section{RESULTS}

The chi-square test did not show any significant difference between the participants in the intervention and control groups in terms of education and occupations. The mean and standard deviation of marriage was $21.15 \pm 4.36$ years in the intervention group and $21.18 \pm 5.47$ in the control group. Independent test results showed that there were no significant differences between the intervention and control groups regarding the mean age and marriage age, and the two groups were homogeneous. Independent tests also showed no significant difference between marriage variables, number of children, age of oldest and youngest child, and income in both the intervention and control groups (Table 2).

Based on the independent test results, there was a statistically significant difference between the mean scores of knowledge, perceived be-
Table 2. Mean age, weight, height, and body mass index in two groups of education and control before education

\begin{tabular}{lccc}
\hline \multicolumn{1}{c}{ Variable } & Control & Education & P-value \\
\hline Age $(\mathrm{y})$ & $10.77 \pm 26.35$ & $10.53 \pm 25.75$ & 0.802 \\
Marriage age $(\mathrm{y})$ & $5.47 \pm 22.18$ & $4.56 \pm 22.15$ & 0.984 \\
Marriage duration $(\mathrm{y})$ & $4.60 \pm 4.17$ & $1.72 \pm 3.60$ & 0.881 \\
No. of children & $1.61 \pm 1.60$ & $1.60 \pm 1.40$ & 0.941 \\
Income status (rials) & $8,965,000 \pm 2,521,850$ & $8,865,000 \pm 2,521,850$ & 0.551
\end{tabular}

Values are presented as mean \pm standard deviation.

havioral control, behavioral intention categories and iron use between the intervention group and the control group on the post-intervention questionnaires $(\mathrm{P}<0.05)$. However, in the case of the subjective norms variable, the t-test showed no significant difference between the scores pre- and post- intervention for the intervention group. The paired ttest for the intervention group showed a significant difference between mean serum ferritin levels just before and 3 months after the intervention (Table 3).

\section{DISCUSSION}

The results of this study showed that through education based on the theory of planned behavior, which emphasizes the main psychological factors of behavioral change, it is possible to increase the iron supplement intake for pregnant women. Accordingly, when women get adequate knowledge about the effects of iron supplementation and are presented with a positive attitude toward such supplementation, they will be determined to use iron supplementation.

Evaluation of the impact of the education intervention on participants' knowledge indicated an overall improvement in iron supplementation scores. In some studies, training has been effective in raising awareness as well as reducing the severity of iron deficiency ane- 
Table 3.The comparison of the average of studied variables in groups of education and control, before and after intervention

\begin{tabular}{|c|c|c|c|c|c|c|c|c|}
\hline \multirow{2}{*}{ Variable } & \multicolumn{3}{|c|}{ Control group } & \multicolumn{3}{|c|}{ Education group } & \multicolumn{2}{|c|}{ Test } \\
\hline & P-value & After & Before & P-value & After & Before & P-value & t-value \\
\hline Knowledge & 0.090 & $3.08 \pm 16.72$ & $3.19 \pm 16.47$ & 0.001 & $18.55 \pm 1.89$ & $16.25 \pm 2.92$ & 0.002 & 3.49 \\
\hline Attitude & 0.620 & $15.98 \pm 22.80$ & $6.46 \pm 21.70$ & 0.020 & $15.34 \pm 29.35$ & $5.92 \pm 23.32$ & 0.020 & 2.17 \\
\hline Subjective norms & 0.930 & $8.84 \pm 18.97$ & $9.35 \pm 19.12$ & 0.270 & $11.54 \pm 19.12$ & $11.77 \pm 16.02$ & 0.924 & 0.06 \\
\hline Perceived behavioral control & 0.904 & $17.00 \pm 9.00$ & $19.00 \pm 19.00$ & 0.001 & $6.00 \pm 24.00$ & $20.50 \pm 17.50$ & 0.001 & 1.45 \\
\hline Intention & 0.960 & $2.17 \pm 3.17$ & $2.44 \pm 3.15$ & 0.029 & $1.87 \pm 4.10$ & $2.42 \pm 3.05$ & 0.010 & 2.020 \\
\hline Behavior & 0.061 & $2.65 \pm 3.00$ & $2.67 \pm 2.75$ & 0.013 & $3.25 \pm 5.45$ & $0.42 \pm 2.25$ & 0.002 & 3.20 \\
\hline Serum ferritin levels & 0.804 & $0.54 \pm 10.60$ & $0.57 \pm 11.61$ & 0.01 & $1.01 \pm 13.61$ & $0.93 \pm 11.56$ & 0.010 & 1.30 \\
\hline
\end{tabular}

Values are presented as mean \pm standard deviation.

mia. ${ }^{13,14)}$ In others, there was little or no knowledge of the effect from education. ${ }^{15,16)}$ In the current study, the changes in attitude scores showed an increase from the elementary to the advanced stages. The increase in attitude scores has been confirmed by Mashoofi et al. ${ }^{17)}$ Mohebi et al. ${ }^{18)}$ showed that educational intervention has a positive effect on increasing the knowledge, attitude, and practices of female students regarding iron deficiency and the anemia that results from it.

Another finding of this study, is the significance of the mean scores of the attitude category pre- and post-intervention. Based on the PRECEDE-PROCEED model, education has been shown to be effective in improving the attitude of mothers about iron deficiency anemia. ${ }^{19)}$ However, education did not produce any statistically significant changes in the subjective norms scores of the intervention group. These results are consistent with the studies of Ahmadi et al. ${ }^{20)}$ Pawlak et al. ${ }^{21)}$ has shown that subjective norms have the most predictive power among the categories. Ahmadi et al. ${ }^{20}$ found in their own study that the mean scores of the subjective norm of their case group decreased significantly after an educational intervention, which is consistent with the findings of this study. Changes in the perceived behavioral control scores also had an increasing trend. ${ }^{17)}$ According to this, if pregnant women feel that environmental factors such as facilities and barriers are under their control, they will increase their consumption of the iron supplement. ${ }^{21)}$ Associations with social support groups may also provide positive effects and promote the consumption of iron supplements. Friends and spouses played an important role in the subjective norms category. Similar studies have also found this to be the case. ${ }^{22,23)}$ The educational intervention had no meaningful statistical effect on the score of subjective norms for the intervention group. These results are also consistent with the studies of Conner et al. ${ }^{24)}$ Thus, it may be necessary to spend more time in training to increase the score of subjective norms. In present study, intention was significantly.

Increased consumption of iron supplements plays an important role in the prevention of maternal and infant morbidity, and the results of this study showed that this supplement intake increased after training. Additionally, there was a significant difference between the amount of iron supplements taken by women before and after training. This shows the importance of the role of education in promoting the intake of iron supplements by pregnant women. ${ }^{25)}$ In this study, training on the level of ferritin has been effective, which has led to a decrease in iron deficiency. Similarly, in a study of PRECEDE-based training, anemia was reduced in children aged 1 to 5 years old. ${ }^{19}$ Therefore, it is suggested that based on the results of the current study, the use of iron supplementation training should be examined and compared with other health education and health promotion theories.

The findings of this research should also be provided to health planners to help prevent the occurrence of complications due to iron deficiency anemia through the implementation of educational programs. Also, the midwives who have a significant caseload of women in hospitals and health centers should use the results of this research to inform their health education and counseling activities as well.

\section{CONFLICT OF INTEREST}

No potential conflict of interest relevant to this article was reported.

\section{ACKNOWLEDGMENTS}

Our thanks are extended to all pregnant women.

\section{REFERENCES}

1. Mirzaie F, Eftekhari N, Goldozeian S, Mahdavinia J. Prevalence of anemia risk factors in pregnant women in Kerman, Iran. Int J Reprod BioMed 2010;8.

2. KHoshfetrat M, Hosseini M, Saneian H. Effectiveness of iron supplementation (6-and 12-weeks) on hematological parameters among non-anemic iron deficient female students. J Shahrekord Univ Med Sci 2012;13:1-11.

3. Allen LH. Anemia and iron deficiency: effects on pregnancy outcome. Am J Clin Nutr 2000;71(5 Suppl):1280S-4S.

4. Bodnar LM, Scanlon KS, Freedman DS, Siega-Riz AM, Cogswell ME. High prevalence of postpartum anemia among low-income women in the United States. Am J Obstet Gynecol 2001;185:438-43.

5. Borna S, Borna H, Ghanbari Z, Khezrdoost S. Anemia and factors that affect it in pregnancy. Tehran Univ Med J 2005;63:448-52.

6. Esmat B, Mohammad R, Behnam S, Shahrzad M, Soodabeh T, Minoo A, et al. Prevalence of iron deficiency anemia among Iranian pregnant women; a systematic review and meta-analysis. J Reprod Infertil 2010; 
11:17-24.

7. Bayoumeu F, Subiran-Buisset C, Baka NE, Legagneur H, MonnierBarbarino P, Laxenaire MC. Iron therapy in iron deficiency anemia in pregnancy: intravenous route versus oral route. Am J Obstet Gynecol 2002;186:518-22.

8. Godin G, Gagne C, Maziade J, Moreault L, Beaulieu D, Morel S. Breast cancer: the intention to have a mammography and a clinical breast examination-application of the theory of planned behavior. Psychol Health 2001;16:423-41.

9. Ajzen I. Perceived behavioral control, self-efficacy, locus of control, and the theory of planned behavior 1. J Appl Soc Psychol 2002;32:66583.

10. Ajzen I. The theory of planned behaviour: reactions and reflections. Psychol Health 2011;26:1113-27.

11. Glanz K, Bishop DB. The role of behavioral science theory in development and implementation of public health interventions. Annu Rev Public Health 2010;31:399-418.

12. Kadam P, Bhalerao S. Sample size calculation. Int J Ayurveda Res 2010;1:55-7.

13. Gielen AC, McDonald EM, Gary TL, Bone LR. Using the precede-proceed model to apply health behavior theories. In: Glanz K, Rimer BK, Viswanath K, editors. Health behavior and health education: theory, research, and practice. 4th ed. Hoboken (NJ): John Wiley \& Sons Inc.; 2008. p. 407-29.

14. Chang S, Zeng L, Brouwer ID, Kok FJ, Yan H. Effect of iron deficiency anemia in pregnancy on child mental development in rural China. Pediatrics 2013;131:e755-63.

15. Jarrah SS, Halabi JO, Bond AE, Abegglen J. Iron deficiency anemia (IDA) perceptions and dietary iron intake among young women and pregnant women in Jordan. J Transcult Nurs 2007;18:19-27.

16. Beard JL, Hendricks MK, Perez EM, Murray-Kolb LE, Berg A, VernonFeagans L, et al. Maternal iron deficiency anemia affects postpartum emotions and cognition. J Nutr 2005;135:267-72.
17. Mashoofi M, Hosseini MM, Wakili Z, Mahmoodi Keli M, Shahrivar F. The effect of education on knowledge, attitude \& practice of midschool girls on iron-deficiency anemia in Khalkhal in 2009. J Health 2010;1:57-66.

18. Mohebi S, Sharifirad GH, Shahsiah M, Botlani S, Matlabi M, Rezaeian M. The effect of assertiveness training on student's academic anxiety. J Pak Med Assoc 2012;62(3 Suppl 2):S37-41.

19. Hazavehei SM, Jalili Z, Heydarnia AR, Faghihzadeh S. Application of the PRECEDE model for controlling iron-deficiency anemia among children aged 1-5, Kerman, Iran. Promot Educ 2006;13:173-7.

20. Ahmadi TS, Taghdisi MH, Nakhaei N, Balali F. Effect of educational intervention based on the theory of planned behaviour on the physical activities of Kerman health center's staff (2008). J Babol Univ Med Sci (JBUMS) 2010;12:62-9.

21. Pawlak R, Connell C, Brown D, Meyer MK, Yadrick K. Predictors of multivitamin supplement use among African-American female students: a prospective study utilizing the theory of planned behavior. Ethn Dis 2005; 15:540-7.

22. Kothe EJ, Mullan BA, Butow P. Promoting fruit and vegetable consumption: testing an intervention based on the theory of planned behaviour. Appetite 2012;58:997-1004.

23. Prelip M, Slusser W, Thai CL, Kinsler J, Erausquin JT. Effects of a school-based nutrition program diffused throughout a large urban community on attitudes, beliefs, and behaviors related to fruit and vegetable consumption. J Sch Health 2011;81:520-9.

24. Conner M, Kirk SF, Cade JE, Barrett JH. Why do women use dietary supplements?: the use of the theory of planned behaviour to explore beliefs about their use. Soc Sci Med 2001;52:621-33.

25. Shahnazi H, Tabar IM, Azarbin S, Hassanzadeh A, Charkazi A, Moodi M. Impact of education based on precede model on knowledge, attitude and behavior of grade two guidance school girls regarding Iron Deficiency Anemia (IDA) in Isfahan, Iran. Health Syst Res 2012;8:77381. 